\title{
Wheatgrass Juice
}

National Cancer Institute

\section{Source}

National Cancer Institute. Wheatgrass Juice. NCI Thesaurus. Code C113791.

The juice extracted from the mature sprouts of wheatgrass, Triticum aestivum, which is a member of the Poaceae family, with potential anti-inflammatory, immunomodulating and chemopreventive activities. Wheatgrass juice contains many vitamins, including $A, B$ vitamins, $\mathrm{C}$ and $\mathrm{E}$, minerals, including selenium, iron, magnesium, calcium, mang anese, copper and zinc, amino acids, chlorophyll and a number of antioxidant enzymes, including superoxide dismutase and cytochrome oxidase. Although the exact mechanism(s) of action through which wheatgrass juice exerts its effect(s) has yet to be fully elucidated, the components in the juice may scavenge free radicals, reduce chemotherapy-induced myelotoxicity, neutralize toxins and carcinogens and modulate the levels of certain proinflammatory cytokines, such as interleukin (IL)- 6, IL-8, IL-10 and IL-12. 